\title{
Studies of the aetiology of neonatal hepatitis and biliary atresia
}

\author{
D. M. DANKS, P. E. CAMPBELL, I. JACK, J. ROGERS, AND A. L. SMITH
}

From the Genetics Research Unit, Royal Children's Hospital Research Foundation; Department of Pathology, Royal Children's Hospital, Melbourne; and Department of Paediatrics, University of Melbourne, Australia

SUMMARY Aetiological factors were sought prospectively in 55 babies with extrahepatic biliary atresia, in 105 with neonatal hepatitis, and in 11 with intrahepatic biliary atresia, seen as a result of nearly complete ascertainment of these conditions in the State of Victoria between 1963 and 1974. In neonatal hepatitis infective causes were shown in 22 babies, galactosaemia in 6 , and $\alpha_{1}$-antitrypsin deficiency in 8; familial occurrence was noted in 10 further babies and unrelated second diseases were present in 24 of the remaining 59 babies. The only clues to aetiology in extrahepatic biliary atresia were a suspicion of time-space clusters, a deficiency of affected babies born to young primiparous women, and an unexpected number of spontaneous abortions in the histories given by the mothers. Genetic factors appeared to be important in intrahepatic biliary atresia, but are not reported in detail.

Hypotheses for the aetiology of neonatal hepatitis and of extrahepatic biliary atresia are presented. Both are considered syndromes with multiple causes. Recurrence risks in sibs are discussed, and are 1 in 7 for neonatal hepatitis of unknown cause, negligible in extrahepatic biliary atresia, and usually 1 in 2 or 1 in 4 in intrahepatic biliary atresia, depending upon the family history.

Neonatal hepatitis was first distinguished from biliary atresia by Craig and Landing in 1952. Intrahepatic biliary atresia was recognized later. The aetiology of all 3 conditions remains ill-defined. A prospective study was started in Melbourne in July 1963 and has included nearly all cases born in Victoria since. The aetiological factors discovered in 55 babies with extrahepatic biliary atresia (EHBA), in 11 with intrahepatic biliary atresia (IHBA), and in 105 with neonatal hepatitis (NNH) seen in the $11 \frac{1}{2}$-year period up to December 1974 are reported.

\section{Definitions}

EHBA. Atresia of part or all of the extrahepatic bile duct system (including right and left hepatic ducts) causing complete obstruction of bile flow. EHBA was proved by laparotomy, cholangiography, and subsequent follow-up. Every inoperable case was followed to death with necropsy examination in all but 3 patients. Those who have drained bile after some form of anastomotic surgery have been followed since (Danks et al., 1974).

Received 31 August 1976
IHBA. A curious disease in which the late anatomical features (after 1 year of age) comprise a fine patent extrahepatic bile duct system and absence, or extreme reduction, of the interlobular ducts normally seen in the portal tracts (Alagille et al., 1975). It is sometimes called biliary hypoplasia (Longmire, 1964). The early microscopical features may be indistinguishable from NNH. Patency of the major ducts was established by clearing of jaundice, by cholangiography, or at necropsy. A distinction was made from NNH by follow-up.

NNH. This was defined by microscopical changes in the liver in 88 babies. The remaining 17 babies were accepted because of jaundice of an obstructive type lasting more than 4 weeks. Prompt demonstration of the aetiology was the reason for not performing a biopsy in many of these babies. Most patients were jaundiced and onset before 4 weeks was demanded. Anicteric babies presenting with steatorrhoea or hypoprothrombinaemia were accepted up to 6 weeks of age. Patency of the bile ducts was established by clearing of jaundice, at laparotomy or at necropsy.

The microscopical features of NNH have been described fully elsewhere (Smith et al., 1977). 
The key features are small foci of liver cell necrosis with cellular infiltration and condensation of reticulin, distortion of the parenchymal cell arrangement by variability in cell size and shape with bi- and trinucleate cells, or multinucleate giant cells, accompanied only by mild inflammation and duct proliferation in the portal tracts.

\section{Methods}

Ascertainment. Careful assessment has shown that all cases of EHBA in Victoria were included in the study and over $80 \%$ of all clinically apparent cases of NNH and IHBA. The criteria used to define NNH have excluded mild cases with transient jaundice or late onset of symptoms. A few additional cases were referred from other States, but these were excluded when calculating the incidence of the conditions.

Analysis of time-space distribution of cases. Analysis was confined to patients with EHBA and to nonfamilial cases of NNH born in metropolitan Melbourne. The patients were listed in chronological sequence of birth dates and their birth places were marked on a scale map of metropolitan Melbourne. The product (time interval in days between birth dates $\times$ distances in miles between birth places) was calculated for pairs of cases taken in birth date sequence, and the mean was calculated. This mean was compared with the mean value of this product for all possible pairs of cases, using a ' $t$ ' test. The method is a slight variation on that of Knox (1971). Another chronological list of all cases, rural and urban, was prepared for visual inspection.

Investigations. Virus isolation studies were performed on a throat swab and on one or more urine samples.
Standard procedures were used to grow and identify rubella and cytomegalovirus (CMV) in monkey kidney cell cultures and in human fetal fibroblast cell cultures respectively. CMV complement-fixing antibodies were measured in serum using the RCH strain 234 antigen. Toxoplasma serology was performed by complement fixation and/or by immunofluorescence. Hepatitis B surface antigen was sought by solid phase radioimmunoassay and antibody by radioimmune precipitation (Dr. Ian Gust, Fairfield Hospital for Communicable Diseases).

A screening test (Beutler and Baluda, 1968) was used to exclude galactosaemia and positive diagnoses were confirmed by measurement of hexose-1-phosphate uridyl transferase activity in red blood cells. Urine was tested for sugar with Clinitest (Ames Co.) with paper chromatography of samples containing sugar. Aminoaciduria was detected by two-dimension paper chromatography until 1971, and by high voltage electrophoresis on paper since then.

$\alpha_{1}$-antitrypsin $\left(\alpha_{1} \mathrm{AT}\right)$ deficiency was detected by measuring the trypsin inhibiting capacity of serum using benzoyl arginine p-nitroanilide hydrochloride as substrate (Eriksson, 1965) until 1970 and by radial immunodiffusion (Behringwerke plates) since. Samples with low levels and samples from relevant family members were subsequently $\mathbf{P i}$ typed (by Dr. Magne Fagerhol of Oslo). All other measurements were made by standard laboratory methods.

\section{Results}

Infective agents. The results of virus studies and serological tests for syphilis and toxoplasmosis are set out in Table 1. CMV can certainly be regarded as causative in 7 babies with $\mathrm{NNH}$ and was probably the cause in $13(15 \%)$. It was also

Table 1 Role of infective agents in aetiology

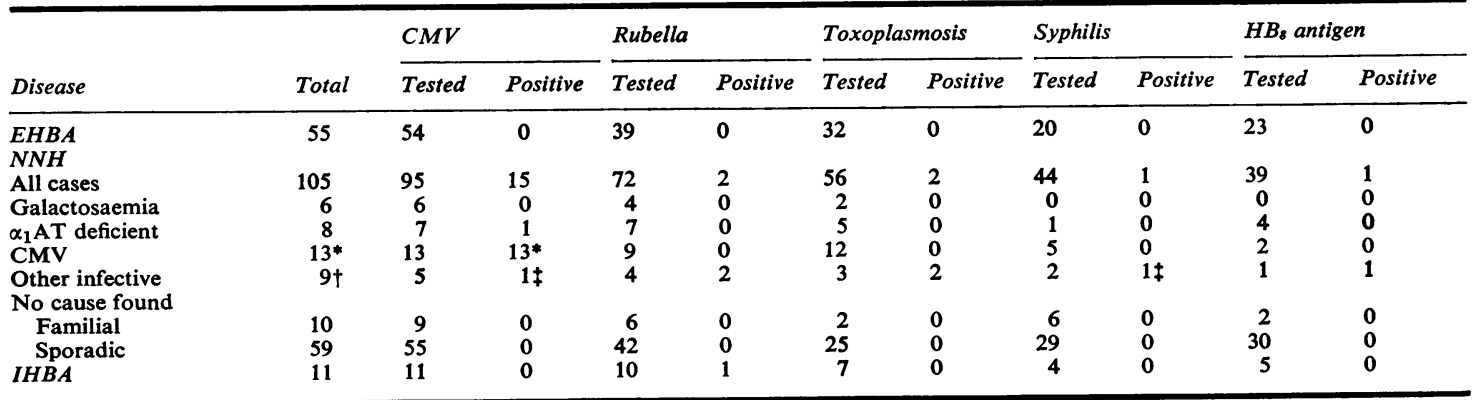

*Virus isolated before 4 weeks in 7 cases, at 4-8 weeks in 6 (see text).

+Includes 1 patient with Coxsackie $B_{2}$ infection, 1 Coxsackie B4, and 1 parainfluenza type 3.

$¥$ This patient had congenital syphilis and also CMV infection.

EHBA = extrahepatic biliary atresia; IHBA $=$ intrahepatic biliary atresia; $N$ NH = aeonatal hepatitis; $\alpha_{1}$ AT $=$ alpha-1-antitrypsin. 
isolated from 1 baby with congenital syphilis and 1 with $\alpha_{1}$ AT deficiency. In 3 patients ( 2 EHBA) initial CMV studies were negative and subsequent samples contained the virus. These were regarded as instances of postnatal infection with this agent.

Rubella virus was isolated from throat and/or urine of 2 babies with NNH, and from 1 with IHBA. The baby girl with IHBA had pulmonary artery stenosis, retinopathy, and osteopathy, and the rubella virus was regarded as the cause of her disease. However, the subsequent birth of an identically affected brother cast serious doubt on this interpretation. One baby with positive serology for toxoplasmosis died of gross cerebral destruction. The other was only mildly affected.

Viruses were isolated from multiple tissues of 3 babies who died of illnesses resembling septicaemia with jaundice, haemorrhages, and hepatosplenomegaly. The agents were Coxsackie $B_{2}$, Coxsackie $B_{4}$, and parainfluenza type 3 respectively. The only baby with HB surface antigen and antibody was born to an opiate addict.

Galactosaemia and galactosuria. 6 babies who presented with a clinical problem of NNH proved to have galactosaemia. Galactosuria of a heavy and persistent type was encountered in 2 babies with NNH who had normal levels of hexose-1phosphate uridyl transferase in their red cells. Both babies were quite ill and were treated with lactose-free feeds with marked clinical improvement shortly after the change in feeding. Subsequent reintroduction of lactose caused no ill effects. This was interpreted as galactose intolerance secondary to liver disease of another cause.

$\alpha_{1}$ AT deficiency. Definite deficiency was shown and confirmed by finding $\mathrm{Pi} \mathrm{ZZ}$ phenotypes in 6 babies with NNH out of 54 tested. 2 other babies with levels low enough to indicate homozygous $\alpha_{1} \mathrm{AT}$ deficiency were unavailable for Pi typing. Homozygous $\alpha_{1} \mathrm{AT}$ deficiency was excluded in 25 babies with EHBA and 9 with IHBA.

Incidence of diseases. Among 790385 children born in Victoria during the study period, 55 had EHBA (6.96/100 000 live births, or 1 in 14000 approximately), 99 had NNH $(12 \cdot 53 / 100000$ live births, 1 in 8000 approximately), and 11 had IHBA $(1 \cdot 39 / 100000$ live births, or 1 in 70000 approximately). The total incidence of neonatal liver disease was 1 in 5000 live births.

Maternal age and birth order. The distribution of maternal age and number of previous live births for patients with each disease was compared with the distribution of these parameters for all live births in Victoria in the same years (Demography, 1963-1973). Tables were not available for 1973 or 1974, and 1972 figures were used for babies born in these years. No significant deviation from expectation was observed for NNH. However, maternal age $(0.001<P<0.01)$ had a strong positive effect upon the occurrence of EHBA. Birth order showed a less striking effect $(0.02<P<0.05)$. However, the data in Table 2 show a striking deficiency of first-born babies of young mothers rather than an excess of babies born to older mothers. Miscarriages and stillbirths were excluded from the calculations and the EHBA mothers had a remarkable number of miscarriages (Table 3), most of which occurred before the birth of the affected child. The total number of previous pregnancies would have been much larger than the figures in Table 2 show.

Familial occurrence. The data in Table 3 were recorded at interviews of both parents (whenever possible) by one of two authors. Information regarding aunts, uncles, and cousins was incomplete in some families. Illnesses in relatives were confirmed from hospitals or doctors in nearly all instances.

The most definite finding was the occurrence of NNH in sibs. Correction for ascertainment bias was achieved by counting the sibs of index cases and by including each family once for each index case giving 12 affected among 81 sibs, or a proportion $0 \cdot 149 \pm 0.040$ affected. This figure is lower than the $0 \cdot 250$ expected in recessive inheritance. NNH can be excluded as the cause of 6 of the 8 neonatal deaths among the sibs of index cases (Table 3), but remains a possibility in the other 2 . One baby had the syndrome of persistent cholestatic jaundice and lymphoedema described by Aagenaes et al. (1968; Aagenaes, 1974). A sib of this patient died in the newborn period without defined cause.

All the affected sibs were male, though the index case was female in one of these sibships. The pedigrees provided no real support for X-linked recessive inheritance. No familial occurrence of EHBA was encountered. The remarkable number of miscarriages in these families has been mentioned.

All the babies with IHBA had unusual facies (Alagille et al., 1975), and 8 had pulmonary artery stenosis, pulmonary valve stenosis, or pulmonary atresia. Among the 11 index cases were a brother and sister each with the full syndrome including severe pulmonary artery stenosis and hypoplasia. One of these babies had also suffered intrauterine infection with rubella (see above). Two maternal uncles of another child were affected by IHBA and the mother and child had a similar unusual 
Table 2 Maternal age and birth rank for patients with NNH of unknown aetiology and with EHBA

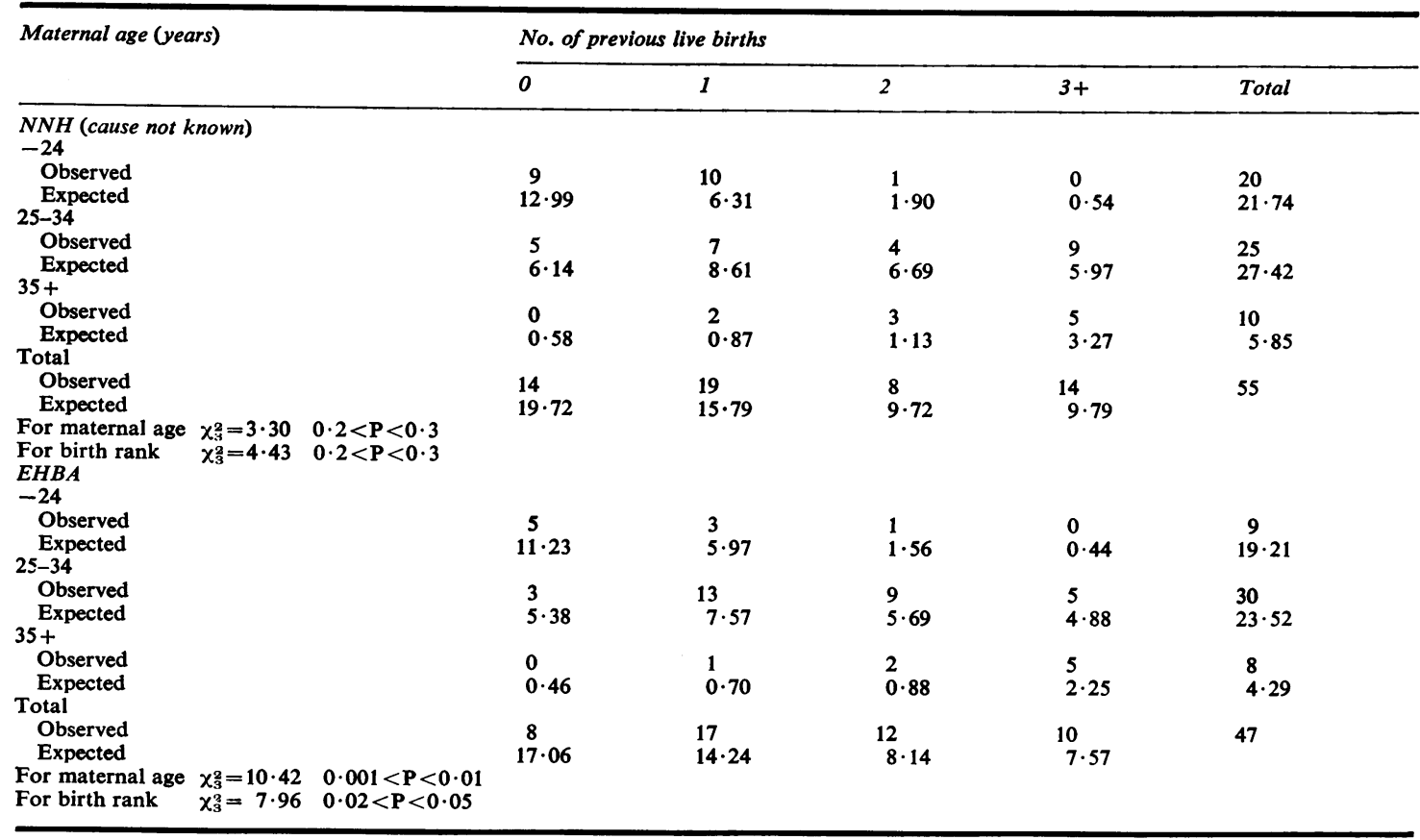

Table 3 Data on relatives of index cases

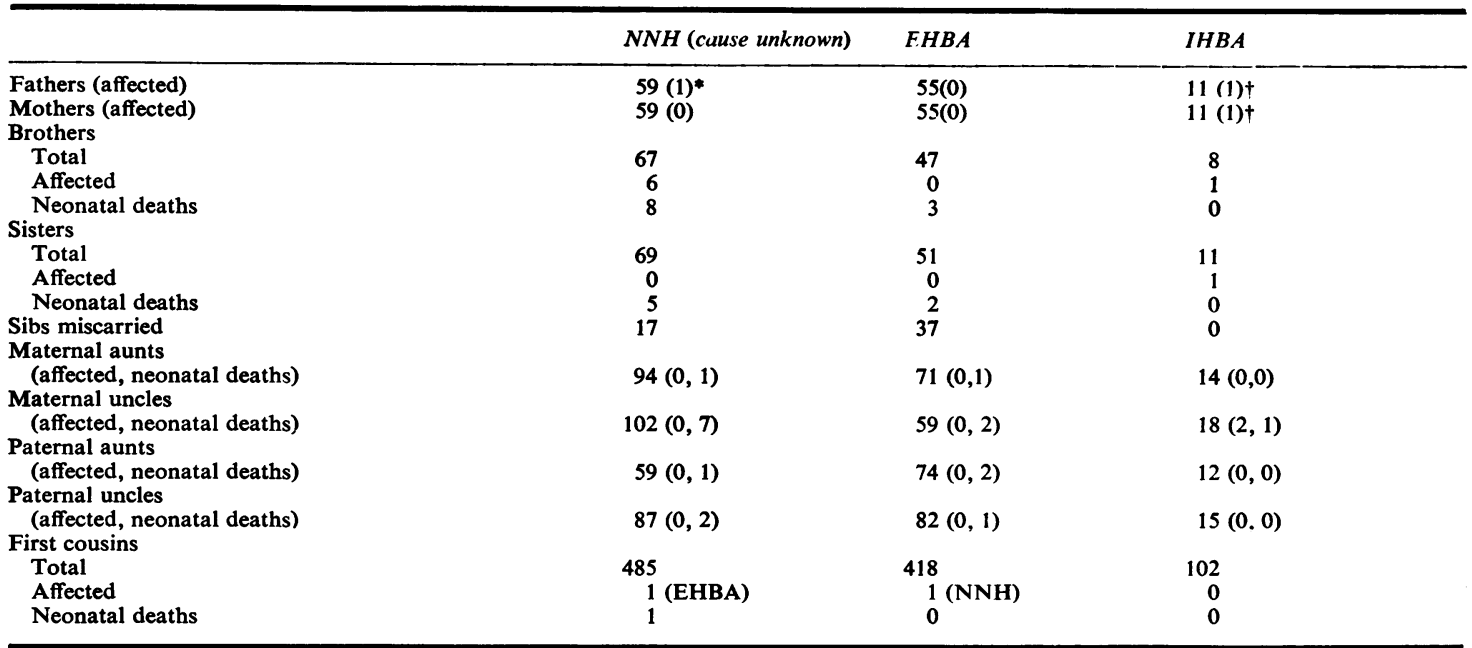

*Jaundice reputedly present for 6 months after birth.

+1 mother and 1 father (from different families) had the characteristic facies without any abnormal hepatic or cardiac findings.

facies. However, the mother give no history of liver disease in childhood and had no cardiac murmur. The father of another baby had the characteristic facies, but no other features.
Time-space distribution of cases. The time sequence of births of patients with NNH of unknown cause was fairly even, averaging 5 cases per year in Victoria. However, two periods of 18 months to 
2 years elapsed without a case of EHBA and two 12-month periods yielded 9 and 12 cases respectively, compared to the annual average of 5 cases per year. Consideration of place of birth increased this suspicion of time-space clustering. For instance, Geelong, the second city of Victoria, produced the anticipated number of cases in the whole of 12 years; however, the 6 patients were born in 2 clusters of 6 months each. A small country town (population 10000 ) produced 2 cases in 3 months. Two neighbouring outer suburbs of Melbourne contributed 4 cases in 4 months.

The extreme differences in population density in urban and rural Victoria have thwarted attempts to devise a meaningful index of spatial dispersion appropriate to both. Analysis of urban cases only showed a significant departure from random timespace distribution $(\mathrm{P}<0.05)$ for EHBA, but not for NNH.

Sex ratio. The sex ratio in the 3 diseases and the sex ratios in each aetiological category of NNH are presented in Table 4 . There was a significant

Table 4 Sex ratio

\begin{tabular}{lcccl}
\hline Disease category & Male & Female & Total & $\begin{array}{l}\text { Sex ratio } \\
(M / F)\end{array}$ \\
\hline EHBA & 27 & 28 & 55 & 0.96 \\
IHBA & 7 & 4 & 11 & $1 \cdot 75$ \\
NNH-all cases & 68 & 37 & 105 & $1 \cdot 84$ \\
NNH-infective cause & 11 & 11 & 22 & $1 \cdot 00$ \\
NNH-genetic cause & 8 & 6 & 14 & $1 \cdot 33$ \\
NNH-familial & 8 & 2 & 10 & $4 \cdot 00$ \\
NNH- & & 18 & 59 & $2 \cdot 28$ \\
\hline
\end{tabular}

excess of males with NNH of unknown aetiology, and among the familial cases of NNH.

Consanguinity. There was only one consanguineous mating, between Italian first cousins, who produced 2 babies who died of NNH. The first died at 4 months after an illness marked by chronic haemolytic anaemia and progressive cerebral degeneration in addition to the liver disease. The second died a few hours after birth and the liver disease was diagnosed at necropsy.

Birthweight and gestational age. Birthweight and gestational age of each baby was plotted on curves derived for normal babies in this community (Kitchen, 1968) and the results are presented by centile groupings (Tables 5,6). Probabilities derived by $\chi^{2}$ analysis of these numbers indicated a low birthweight in NNH caused by infective agents, and both low birthweight and premature delivery in babies with NNH of unknown cause.

Maternal illness and medication. This was recorded in a standardized manner for 45 babies with EHBA and 53 of the babies with NNH of unknown aetiology. No control group was available but comparison of the two diseases showed no difference in the frequency or nature of illnesses or complications during pregnancy. Drug consumption was similar except that iron was taken by 23 mothers of babies with EHBA and by only 9 mothers of babies with NNH.

Second diseases. Second diseases were identified in 24 of the 59 babies with NNH of unknown cause with negative family histories (Table 7). Second diseases were not encountered among babies with NNH of known cause, and were present in only 3 of 58 babies with EHBA. Pulmonary valve and/or pulmonary artery abnormalities and unusual facies have been mentioned already in IHBA.

Bile duct remnants in EHBA. These remnants were studied systematically. The macroscopical appearances at operation were recorded personally by one author (D.M.D.) as thick fibrous cords (14 cases), fine fibrous strands (16 cases), swollen

Table 5 Birthweight according to gestation

\begin{tabular}{|c|c|c|c|c|c|c|c|}
\hline & \multicolumn{6}{|c|}{ Centiles } & \multirow[b]{2}{*}{$\begin{array}{l}\text { Total } \\
\text { known }\end{array}$} \\
\hline & $0-2$ & $3-24$ & $25-49$ & $50-74$ & $75-96$ & $97-100$ & \\
\hline $\begin{array}{l}\text { EHBA } \\
\text { IHBA } \\
\text { NNH-all cases } \\
\text { NNH-infective cause* } \\
\text { NNH-genetic cause } \\
\text { NNH-familial } \\
\text { NNH-cause unknown } \dagger\end{array}$ & $\begin{array}{l}1 \\
0 \\
9 \\
0 \\
2 \\
0 \\
7\end{array}$ & $\begin{array}{r}13 \\
6 \\
29 \\
11 \\
1 \\
3 \\
14\end{array}$ & $\begin{array}{r}9 \\
2 \\
15 \\
3 \\
1 \\
1 \\
10\end{array}$ & $\begin{array}{r}10 \\
1 \\
22 \\
1 \\
4 \\
2 \\
15\end{array}$ & $\begin{array}{r}22 \\
2 \\
17 \\
3 \\
3 \\
3 \\
8\end{array}$ & $\begin{array}{l}0 \\
0 \\
4 \\
0 \\
0 \\
0 \\
4\end{array}$ & $\begin{array}{r}55 \\
11 \\
96 \\
18 \\
11 \\
9 \\
58\end{array}$ \\
\hline
\end{tabular}

* Significant deviation from expectation $(P<0.01)$.

+ Significant deviation from expectation $(0.02<P<0.05)$. 
Table 6 Gestational age

\begin{tabular}{|c|c|c|c|c|c|c|}
\hline \multirow[b]{2}{*}{ Disease category } & \multicolumn{5}{|c|}{ Gestation (w) } & \multirow[b]{2}{*}{ Total } \\
\hline & $<32$ & $32-35$ & $36-37$ & $38-41$ & $42+$ & \\
\hline EHBA & 0 & 5 & 3 & 46 & 1 & 55 \\
\hline IHBA & 0 & $\mathbf{0}$ & 2 & 8 & 0 & 10 \\
\hline NNH-all cases & 4 & 15 & 19 & 56 & 9 & 103 \\
\hline NNH-infective cause & 1 & 2 & 1 & 15 & 2 & 21 \\
\hline NNH-genetic cause & 0 & $\mathbf{0}$ & 1 & 10 & 1 & 12 \\
\hline NNH—familial & $\mathbf{0}$ & 2 & 1 & 5 & 1 & 9 \\
\hline NNH-cause unknown* & 1 & 11 & 16 & 26 & 5 & 59 \\
\hline $\begin{array}{l}\text { Normal population } \dagger \\
\%\end{array}$ & $\begin{array}{l}47 \\
(2 \cdot 34)\end{array}$ & $\begin{array}{l}82 \\
(4 \cdot 08)\end{array}$ & $\begin{array}{l}135 \\
(6 \cdot 72)\end{array}$ & $\begin{array}{l}1520 \\
(75 \cdot 66)\end{array}$ & $\begin{array}{l}225 \\
(11 \cdot 20)\end{array}$ & $\begin{array}{l}2009 \\
(100)\end{array}$ \\
\hline
\end{tabular}

*Deviation from population figure very highly significant $(\mathrm{P}<0 \cdot 0001)$.

†Figures of Kitchen (1968).

Table 7 Second diseases present in the 59 babies with NNH of unknown aetiology (sporadic cases)

\begin{tabular}{ll}
\hline Down's syndrome & 4 \\
Haemolytic disease of newborn (Rh & \\
$\quad$ incompatibility) & 6 \\
Chondrodysplasia punctata & 3 \\
Generalized fibromatosis & 2 \\
Cystic fibrosis & 2 \\
Cystinosis & 1 \\
Niemann-Pick disease & 1 \\
Hepatic haemangioendotheliomatosis & 1 \\
Polycystic disease of kidneys & 1 \\
Hydrocephalus & 1 \\
Congenital heart defect & 1 \\
Multiple congenital malformations & 1 \\
Total & 24 \\
\hline
\end{tabular}

red oedematous cord ( 1 case), no remnants ( 17 cases), not explored, or not adequately recorded ( 7 cases).

Microscopical sections of fibrous remnants removed at operation were examined in 12 patients and the findings were as described by Kasai (1974), i.e. whorls of fibrous tissue around islands of duct epithelium with some inflammatory cells.

Postnatal development of EHBA. In 6 babies completely acholic stools developed only some weeks or months after the onset of jaundice. The bile-containing stools passed by these babies had been seen repeatedly by the authors. The initial needle liver biopsies showed changes strongly suggestive of NNH in each of these babies, but the microscopical feature of mechanical obstruction to bile ducts developed later. The acutely inflamed bile duct described above was found in one of these babies.

\section{Discussion}

Neonatal hepatitis. The broad definition of NNH used in this article inevitably means that multiple aetiologies will be found. However, any more restrictive definition is even less satisfactory. Definable intrauterine infections were responsible for approximately $20 \%$ of NNH (CMV $12 \%$, toxoplasmosis $2 \%$, other agents $6 \%$ ). The rigid criteria used may have underestimated the role of CMV, but occurrence of early postnatal CMV infection creates a dilemma which cannot be resolved satisfactorily. Galactosaemia accounted for 5\% of cases but hereditary fructose intolerance and hereditary tyrosinaemia were not encountered. Unfortunately the data on $\alpha_{1} \mathrm{AT}$ deficiency was incomplete-a figure between 6 out of $54(11 \%)$ and 8 out of $54(15 \%)$ can be estimated, and agrees with published figures.

The patients with NNH in whom all aetiological tests proved negative offer the most interesting challenge. A number of characteristics of this group can be described-premature delivery, low birthweight for maturity, striking excess of males, occurrence in sibs with a frequency of about $15 \%$, and occurrence of a second disease in $40 \%$ of those patients who have no affected sibs. This group is almost certainly heterogeneous. Recessively inherited conditions seem certain in at least 3 families - the consanguineous couple who produced 2 affected babies, another couple who produced 4 healthy girls and 3 sons who died of acute liver failure at about 1 week of age (Danks, 1974), and the baby with Aagenaes's syndrome (1974). Other recessively inherited conditions may exist in this group.

A different hypothesis is advanced to explain the majority of cases of NNH. We suggest that transplacental infection by one of many infective agents capable of damaging the liver is fairly common (e.g. occurs in $5-10 \%$ of pregnancies) but generally causes no noticeable illness, and that the various second diseases encountered in this study predispose some fetuses to develop overt disease. Unrecognized genetic factors may act in this way, and so may $\alpha_{1}$ AT deficiency. Many of the second diseases noted are known to be associated with increased susceptibility to infection (e.g. Down's syndrome, chondrodysplasia punctata), and others cause some 
liver damage (cystic fibrosis, Niemann-Pick disease), or place an unusual load on liver function (e.g. haemolytic disease of the newborn).

The circumstances encountered in haemolytic disease of the newborn (HDN) provide some support for this hypothesis. When needle liver biopsy has been performed in babies with HDN and persistently raised conjugated bilirubin in the serum ('inspissated bile syndrome'), it has always shown changes of mild NNH. HDN subjects the liver to a bilirubin load, and might cause jaundice in babies with subclinical NNH. These patients should have a good prognosis-this is true. The frequency of the 'inspissated bile syndrome' in HDN should give an estimate of the frequency of subclinical NNH in the newborn. This is the origin of the $5-10 \%$ figure suggested above. This figure should also indicate the proportion of pregnant women harbouring agents capable of causing hepatitis and should correspond to the frequency of subclinical hepatitis after blood transfusion-also 5-10\% (Hampers et al., 1964; Creutzfeldt et al., 1966; Proskey et al., 1970). This figure is much higher than the frequency of HBs antigenaemia, and other agents must be proposed in addition to the hepatitis B (Knodell et al., 1975). Intrauterine transfusions in severe HDN should raise the frequency of the 'inspissated bile syndrome' by exposing the fetus to donor blood in addition to the mother-this is also true.

Many reports describe the occurrence of NNH in babies with other serious diseases of infancy, e.g. trisomy E (Alpert et al., 1969), Niemann-Pick disease (Ivemark et al., 1963; Ashkenazi et al., 1971). It seems more reasonable to propose that most of the diseases listed in Table 7 act by nonspecifically increasing susceptibility to agents capable of causing hepatitis than to claim that each can specifically cause a liver lesion in an occasional baby. The baby with polycystic kidneys is of interest in this regard. A subsequent sib was born with identical polycystic kidneys and bile duct dysplasia, but did not have clinical or microscopical evidence of NNH.

Extrahepatic biliary atresia. In EHBA there was no familial occurrence, the birthweight and sex ratio were unremarkable, and there was suspicion (but not proof) of time-space clustering. The existence of inflammatory changes in the fibrous duct remnants was an important feature.

The following hypothesis is advanced for the aetiology of EHBA.

(1) Most cases of EHBA are the result of inflammatory destruction of previously formed bile ducts during a cholangitic hepatitis. (2) The hepatitis is probably caused by an agent or agents different from those involved in NNH-probably one or several uncommon agents each causing disease in most fetuses infected. This suggestion is made to explain the striking differences between EHBA and $\mathrm{NNH}$ with regard to predisposing diseases and familial occurrence.

This hypothesis differs from that of Landing (1974) who suggests a common aetiology for NNH and EHBA. The great differences in the data about the two diseases presented here make this suggestion very unlikely. A single cause for either disease seems improbable, leave alone one cause for both diseases.

No explanation of the excessive frequency of miscarriage in pregnancies conceived before the EHBA babies is apparent.

Intrahepatic biliary atresia. Reports of familial occurrence of IHBA have appeared in recent years (Alagille et al., 1975). Heterogeneity seems probable even within the cases seen in Melbourne. The distribution in 2 families would suggest autosomal dominant inheritance of IHBA, pulmonary stenosis, and a characteristic facies with considerable variation from patient to patient, while autosomal recessive inheritance seems more likely in another family.

Knowledge of aetiology may allow logical treatment eventually and will allow counselling about future pregnancies. The present study and past studies (Hsia et al., 1958; Danks and Bodian, 1963) show that parents of babies with NNH of unknown cause must be given guarded advice-about 1 in 7 of subsequent babies will be affected. In EHBA the risk of a second affected baby must be very low. The larger studies of this condition (Hsia et al., 1958; Danks and Bodian, 1963; present study) contain no family with a second case among a total of 142 families. Several papers have reported individual families claimed to contain 2 or more babies with EHBA (Nevin et al., 1969). However, careful study of the patients reported shows that some date from the period before NNH was distinguished from EHBA and that most of the more recent cases have suffered from the unusual entity of EHBA plus IHBA. Like IHBA, this condition does show a definite familial occurrence.

We acknowledge the assistance of the many people who contributed to this long-term prospective study, especially the referring paediatricians, the surgeons who operated on the babies with biliary atresia, and laboratory staff of the Royal Children's Hospital. Drs. Kathleen Hayes and John Connelly gave particular assistance with the laboratory investigations. 


\section{References}

Aagenaes, Ø. (1974). Hereditary recurrent cholestasis with lymphoedema-two new families. Acta Paediatrica Scandinavica, 63, 465-471.

Aagenaes, Ø., van der Hagen, C. B., and Refsum, S. (1968). Hereditary recurrent intrahepatic cholestasis from birth. Archives of Disease in Childhood, 43, 646-657.

Alagille, D., Odièvre, M., Gautier, M., and Dommergues, J. P. (1975). Hepatic ductular hypoplasia associated with characteristic facies, vertebral malformations, retarded physical, mental and sexual development, and cardiac murmur. Journal of Pediatrics, 86, 63-71.

Alpert, L. I., Strauss, L., and Hirschhorn, K.(1969). Neonatal hepatitis and biliary atresia associated with trisomy 17-18 syndrome. New England Journal of Medicine, 280, 16-20.

Ashkenazi, A., Yarom, R., Gutman, A., Abrahamov, A., and Russell, A. (1971). Niemann-Pick disease and giant cell transformation of the liver. Acta Paediatrica Scandinavica, 60, 285-294.

Beutler, E., and Baluda, M. (1968). A simple spot screening test for galactosemia. Journal of Laboratory and Clinical Medicine, 68, 137-141.

Craig, J. M., and Landing, B. H. (1952). Form of hepatitis in neonatal period simulating biliary atresia. Archives of Pathology, 54, 321-333.

Creutzfeldt, W., Severidt, H.-J., Schmitt, H., Gallasch, E., Arndt, H. J., Brachmann, H., Schmidt, G., and Tschaepe, U. (1966). Incidence and course of icteric and anicteric transfusion hepatitis. German Medical Monthly, 11, 469-476.

Danks, D. M. (1974). Management of newborn babies in whom serious metabolic illness is anticipated. Archives of Disease in Childhood, 49, 576-578.

Danks, D. M., and Bodian, M. (1963). A genetic study of neonatal obstructive jaundice. Archives of Disease in Childhood, 38, 378-387.

Danks, D. M., Campbell, P. E., Clarke, A. M., Jones, P. G., and Solomon, J. R. (1974). Extrahepatic biliary atresia-the frequency of potentially operable cases. American Journal of Diseases of Children, 128, 684-686.

Demography (1963-1973). Commonwealth Bureau of Census and Statistics. Melbourne, Australia.
Eriksson, S. (1965). Studies in alpha ${ }_{1}$-antitrypsin deficiency. Acta Medica Scandinavica, 177, Suppl., 432.

Hampers, C. L., Prager, D., and Senior, J. R. (1964). Post-transfusion anicteric hepatitis. New England Journal of Medicine, 271, 747-754.

Hsia, D. Y.-Y., Boggs, J. D., Driscoll, S. G., and Gellis, S. S. (1958). Prolonged obstructive jaundice in infancy. V. The genetic components in neonatal hepatitis. American Journal of Diseases of Children, 95, 485-491.

Ivemark, B. I., Svennerholm, L., Thorén, C., and Tunell, R. (1963). Niemann-Pick disease in infancy. Report of two siblings with clinical, histologic and chemical studies. Acta Paediatrica Scandinavica, 52, 391-404.

Kasai, M. (1974). Treatment of biliary atresia with special reference to hepatic porto-enterostomy and its modifications. Progress in Pediatric Surgery, 6, 5-52.

Kitchen, W. H. (1968). The relationship between birth weight and gestational age in an Australian hospital population. Australian Paediatric Journal, 4, 29-37.

Knodell, R. G., Conrad, M. E., Dienstag, J. L., and Bell, C. J. (1975). Etiological spectrum of post-transfusion hepatitis. Gastroenterology, 69, 1278-1285.

Knox, E. G. (1971). Epidemics of rare diseases. British Medical Bulletin, 27, 43-47.

Landing, B. H. (1974). Considerations of the pathogenesis of neonatal hepatitis, biliary atresia and choledochal cyst - the concept of infantile obstructive cholangiopathy. Progress in Pediatric Surgery, 6, 113-139.

Longmire, W. P. (1964). Congenital biliary hypoplasia. Annals of Surgery, 159, 335-343.

Nevin, N. C., Bell, M., Frazer, M. J. L., and Froggatt, P. (1969). Congenital extrahepatic biliary atresia in two brothers. Journal of Medical Genetics, 6, 379-381.

Proskey, V. J., Morrison, G. R., McQuillan, B. P., and Parker, B. M. (1970). Anicteric and icteric hepatitis after open-heart surgery. Gastroenterology, 58, 203-207.

Smith, A. L., Danks, D. M., and Campbell, P. E. (1977). The microscopic changes in the liver in neonatal hepatitis and extrahepatic biliary atresia. (Submitted for publication.)

Correspondence to Prof. D. M. Danks, Department of Paediatrics, Royal Children's Hospital, Parkville, Victoria 3052, Australia. 